\title{
Comparison of the outcomes of different human spermatozoa selection methods in assisted reproduction.
}

\author{
Zhanhui Ou, Li Yang, Zhiheng Chen, Yu Deng, Hui Wang, Ling Sun* \\ Center of Reproductive Medicine, Guangzhou Women and Children's Medical Center, Guangzhou Medical University, \\ Guangzhou, PR China
}

\begin{abstract}
Selecting sperm by density-gradients in assisted reproductive technologies has a great improvement in keeping DNA integrity and sperm quality. However, study showed that histone retention and DNA methylation were changed in the method of selecting human spermatozoa by density-gradients in assisted reproductive technologies. In the present study, a total of 1874 cycles (1411cycles of using density-gradients method or the combination of density-gradients and swim-up (group A) and 463 cycles of using swim-up only (group B)) of in vitro fertilization (IVF)/Intracytoplasmic Sperm Injection (ICSI) that were performed in our hospital from January 2010 to December 2015 were retrospectively analyzed. Implantation rate, clinical pregnancy rate, miscarriage rate, ectopic pregnancy rate, twin pregnancy rate, still birth rate, prematurity rate, low birth weight rate, live birth rate, macrosomia rate and fetal or baby abnormality rate were compared. There were no significant differences in the clinical items shown above between two groups except for the live birth rate of per transferred cycle. Live birth rate was higher in the group A than group B. We conclude that both using density-gradients (or combination with swim-up) and swim-up method alone to separate sperm are safe to prepare the sperm for IVF and ICSI.
\end{abstract}

Keywords: Density-gradients, Swim-up, Sperm, Clinical outcomes, Assisted reproductive technologies.

Accepted on May 18, 2018

\section{Introduction}

It is estimated that infertility affects one in six couples worldwide, and about half of these cases are due to male factors [1]. There are many factors that can cause men infertile, but only several well-characterized infertility phenotypes are with known pathologies [2,3]. Studies have demonstrated that common genetic variants are not responsible for the most of male infertility [4].

Transition of histone to protamine is considered as a key step during spermatogenesis [5]. And the exchange rate of histone to protamine is estimated to be about $85 \%$ in human sperm [6]. DNA methylation is an important form of gene regulation during mammalian development. In the whole genome, oocyte DNA tends to be hypo-methylated while sperm DNA tends to be hyper-methylated [7]. Recently, many studies showed that abnormal changes in sperm histone transition rates and global DNA methylation are associated with decreased semen quality, contributed to the male infertility and failed Assisted Reproductive Technology (ART) [8-11].

Although the In Vitro Fertilization (IVF) and Intracytoplasmic Sperm Injection (ICSI) technique have helped these people to conceive successful; there are some concerns that the use of assisted reproductive technology might increase the risk of birth defects. Hansen et al. reported that infants conceived by the assist of IVF or ICSI had a double risk of a major birth defect than the naturally conceived infants [12]. Several studies also had shown that children born with the assist of ART had a higher risk of getting genomic imprinting disorders such as Beckwith-Wiedemann syndrome or Silver-Russell syndrome [13-15]. Moreover, it was reported that the specific alterations in sperm DNA genome like DNA methylation and histone transition could be passed to the next generation [16].

Sperm separation with density-gradients and swim-up method are routinely applied in the clinic work as assisted reproductive technologies. Density-gradients sperm selection can separate sperm cells with high motility and good morphology. However, it was reported by $\mathrm{Yu}$ that epigenetic alterations were found in the human sperm selecting by density-gradients in assisted reproduction, and their study also suggested the possibility that sperm with good density also possess unique epigenetic profiles, particularly for genes involving in neural and olfactory development [17]. But information of the outcomes of different human spermatozoa selection methods for assisted reproduction in clinical work remains limited. So we compared the outcomes of assisted reproductive technologies with using density-gradients (or combination of swim-up method) and swim-up method alone respectively to separate the sperm cells. 


\section{Materials and Methods}

\section{Study design}

A total of 1874 cycles (1411 of density-gradients or combination with swim-up cycles and 463 of swim-up cycles) of in vitro fertilization (IVF)/Intracytoplasmic Sperm Injection (ICSI) fresh transfer cycles performed at Guangzhou Women and Children's Medical Center from January 2010 to December 2015 were retrospectively analyzed. We chose the fresh transfer cycles in order to exclude the effect of frozenthawed on the embryo. The causes of infertility included female factors, male factors, combined factors, and unexplained infertility. As no additional intervention or investigation was included, ethical approval was not required in this study.

\section{Ovarian stimulation and fresh embryo transfer}

Patients undergoing IVF/ICSI cycles were subjected to controlled ovarian stimulation using a GnRH agonist (Decapetyl, Ipsen Pharma Biotech, Signes, France) or antagonist (Cetrotide, Serono, London, UK) for hypolalamic down-regulation; human menopausal (Menopur, Ferring $\mathrm{GmbH}$, Kiel, Germany) or recombinant FSH (Gonal-F, Serono, UK; or Puregon, Organon/Schering-Plough, UK) for ovarian stimulation; and hCG (Ovidrel, Serono, London, UK) for induction of oocyte maturation. After ovarian puncture COCs were incubated in IVF medium (all culture media from Vitorlife, Sweden AB). Embryos were cultured in G1 or G2 medium according to the culture time. Cleaving embryos were scored every day for blastomere number, cleavage plane and degree of fragmentation and graded accordingly. Higher grade embryos were selected for fresh embryo transfer. Injections of $40 \mathrm{mg} / \mathrm{d}$ of progesterone were commenced on the day of ovulation for one day after which the dose was increased to 80 $\mathrm{mg} / \mathrm{d}$.

\section{Sperm preparation}

All semen samples were collected by masturbation ( 3 to $5 \mathrm{~d}$ of ejaculatory abstinence). Density-gradients medium was prepared in a $15 \mathrm{~mL}$ test-tube by layering $1 \mathrm{~mL}$ of $40 \%(\mathrm{v} / \mathrm{v})$ density-gradient medium over $1 \mathrm{~mL}$ of $80 \%(\mathrm{v} / \mathrm{v})$ densitygradients medium (Vitorlife, Sweden AB). All of each wellmixed semen sample was placed on top of the media and then centrifuged at $400 \mathrm{~g}$ for $20 \mathrm{~min}$. Most of the supernatant was removed from the sperm pellet, re-suspended in $5 \mathrm{~mL}$ of supplemented medium (GIVF, Vitorlife, Sweden AB) by gentle pipetting. The separated sperm pellet was centrifuged at $400 \mathrm{~g}$ for $10 \mathrm{~min}$ twice, and the final sperm pellet was swim-up in about $0.2 \mathrm{ml}$ GIVF (group A). Some semen samples were swim-up in about $2 \mathrm{ml}$ GIVF directly as the swim-up method group (group B).

\section{Definition of outcome}

Clinical pregnancies were defined by the observation of a gestational sac with or without a fetal heartbeat on ultrasound evaluation 4 weeks after Embryo Transfer (ET). The number of sacs was taken as the number of successful implantation. Clinical miscarriage was defined when a pregnancy failed to progress after an intra-uterine gestational sac had been detected by pelvic ultrasonography. Live birth rate was defined live birth in per ET. The clinical pregnancy rate, implantation rate, miscarriage rate, ectopic pregnancy rate and live birth data were collected. The neonatal outcomes evaluated were: prematurity (delivery before 37 weeks), low birth-weight (birth-weight $<2500 \mathrm{~g}$ ), macrosomia (birth-weight $>4000 \mathrm{~g}$ ), fetal or neonatal abnormalities.

\section{Statistical analysis}

Statistical analysis was performed by SPSS software (Chicago, IL, USA) version 19 for Windows, applying parametric and nonparametric tests when appropriate. Continuous variables were expressed as absolute numbers, mean \pm standard deviation (SD), and analyzed by Student t-test. Categorical variables were expressed as percentages and analyzed through the chi-square test (Fisher exact test were used depend on the sample size). Statistical significance was defined as $p$ values less than 0.05 .

\section{Results}

\section{Comparison of demographic characteristics}

Between January 2010 and December 2015, a total of 1874 fresh embryo transfers were included in the final analysis. Overall, the mean age of the women was $(32.31 \pm 4.45)$ y. 1411 of cycles used density-gradients or combination with swim-up method (group A) and 463 of cycles used swim-up method to separate sperm (group B). Average patient age was similar for both groups $(32.24 \pm 4.50$ y versus $32.49 \pm 4.30$ y for group A and group B, respectively). Patients' characteristics in the two groups are listed in Table 1. There were no significant differences between the two groups with respect to age, Body Mass Index (BMI) $(21.24 \pm 2.61$ versus $21.68 \pm 3.02)$, basal FSH $(5.92 \pm 2.13$ versus $5.95 \pm 1.99)$ and estradiol (E2) on the 1 st day of HCG $(7973.30 \pm 4162.94$ versus $8295.55 \pm 4240.48)$ level, causes of infertility (primary (47.98\% versus $51.84 \%$ ) and secondary $(52.02 \%$ versus $48.16 \%)$ ), and endometrial thickness $(10.98 \pm 2.74$ versus $10.95 \pm 2.95)$, and number of embryos transferred per-cycle $(1.94 \pm 0.48$ versus $1.85 \pm 0.44)$ (all, $\mathrm{p}>0.05$ ) (Table 1).

\section{Comparison of clinical outcomes}

The clinical outcomes are summarized in Table 2. Despite a similar number of transferred embryos in both groups, the live birth rate per transferred cycle was higher in the group A as compared to the group B $(55.99 \%$ versus $48.60 \%$, respectively, $\mathrm{P}<0.01)$. The rate of twin pregnancies was higher in the group A, but without statistical significance $(29.97 \%$ versus $23.78 \%$, respectively). There were no significant differences between the two groups with respect to implantation rate $(36.15 \%$ versus $35.55 \%)$, clinical pregnancy rate $(52.09 \%$ versus $50.76 \%)$, miscarriage rate $(5.67 \%$ versus $7.78 \%)$, ectopic 
pregnancy rate $(1.63 \%$ versus $2.59 \%)$, and still birth rate $(0.63 \%$ versus $1.32 \%)$ (all, $\mathrm{p}>0.05)$ (Table 2$)$.

Table 1. Baseline patient characteristics.

\begin{tabular}{|c|c|c|c|}
\hline & Group A ( $n=1411)$ & Group B $(n=463)$ & $\mathbf{P}$ \\
\hline Age $(y)$ & $32.24 \pm 4.50$ & $32.49 \pm 4.30$ & NS \\
\hline BMI $\left(\mathrm{kg} / \mathrm{m}^{2}\right)$ & $21.24 \pm 2.61$ & $21.68 \pm 3.02$ & NS \\
\hline Basal FSH (mlU/mL) & $5.92 \pm 2.13$ & $5.95 \pm 1.99$ & NS \\
\hline E2 on the 1st day of HCG (pmol/L) & $7973.30 \pm 4162.94$ & $8295.55 \pm 4240.48$ & NS \\
\hline \multicolumn{4}{|l|}{ Infertility } \\
\hline Primary (\%) & $677(47.98)$ & $240(51.84)$ & NS \\
\hline Secondary (\%) & $734(52.02)$ & $223(48.16)$ & NS \\
\hline Endometrial thickness (mm) & $10.98 \pm 2.74$ & $10.95 \pm 2.95$ & NS \\
\hline No. of embryos transferred & $1.94 \pm 0.48$ & $1.85 \pm 0.44$ & NS \\
\hline
\end{tabular}

BMI: Body Mass Index; E2: Estradiol; FSH: Follicle Stimulating Hormone; Data expressed as mean \pm SD or percentage (\%).

Table 2. Pregnancy outcomes between two groups during IVF/ICSI treatment.

\begin{tabular}{llll}
\hline & $\begin{array}{l}\text { Group } \\
(\mathbf{n = 1 4 1 1 )}\end{array}$ & A $\begin{array}{l}\text { Group } \\
(\mathbf{n = 4 6 3 )}\end{array}$ & B \\
\hline Implantation rate (\%) & $996 / 2755(36.15)$ & $305 / 858(35.55)$ & NS \\
\hline Clinical pregnancy rate (\%) & $735 / 1411(52.09)$ & $235 / 463(50.76)$ & NS \\
\hline Miscarriage rate (\%) & $80 / 1411(5.67)$ & $36 / 463(7.78)$ & NS \\
\hline Ectopic pregnancy rate (\%) & $23 / 1411(1.63)$ & $12 / 463(2.59)$ & NS \\
\hline Still birth (\%) & $5 / 795(0.63)$ & $3 / 228(1.32)$ & NS \\
\hline Live birth rate (\%) & $790 / 1411(55.99)$ & $225 / 463(48.60)$ & $<0.01$ \\
\hline Singleton pregnancy (\%) & $430(70.03)$ & $141(76.21)$ & NS \\
\hline Twin pregnancy (\%) & $184(29.97)$ & $44(23.78)$ & NS \\
\hline
\end{tabular}

The prematurity rate, low birth weight rate, and macrosomia rate in the group $\mathrm{A}$ were all higher than that in the group $\mathrm{B}$
(29.32\% versus $24.86 \%, 25.91 \%$ versus $19.74 \%$, and $2.39 \%$ versus 2.19 , respectively). Fetal or baby abnormalities rate was higher in the group B as compared to the group A $(0.75 \%$ versus $1.31 \%$, respectively); however, these did not reach statistical significance (all, $\mathrm{p}>0.05$ ) (Table 3). Anomalies that generally cause functional impairment or require surgical correction or other congenital malformations were found by ultrasound examination during pregnancy and physical examination after birth were recorded. There were 6 cases of anomaly in the group $\mathrm{A}$, and they were: a) Multiple malformations in the chest, ribs, thoracic vertebrae and tetralogy of Fallot; b) Tetralogy of Fallot; c) Patent ductus arteriosus and patent oval foramen; d) Patent ductus arteriosus and cryptorchidism; 5) Adhesion of labia minora require surgical correction; 6) Severe hydrocephalus. And three anomalies cases in the group B: a) Anus imperforatus; b) Tetralogy of Fallot; c) External ear deformity.

Table 3. Neonatal outcomes between two groups after IVF/ICSI treatment.

\begin{tabular}{|c|c|c|c|}
\hline & Group A $(n=1411)$ & Group B $(n=463)$ & $\mathbf{P}$ \\
\hline Prematurity rate $(\%)$ & $180 / 614(29.32)$ & $46 / 185(24.86)$ & NS \\
\hline Low birth weight rate (\%) & $206 / 795(25.91)$ & $45 / 228(19.74)$ & NS \\
\hline Macrosomia rate (\%) & $19 / 795(2.39)$ & $5 / 228(2.19)$ & NS \\
\hline Fetal or neonatal abnormalities rate $(\%)$ & $6 / 795(0.75)$ & $3 / 228(1.31)$ & NS \\
\hline
\end{tabular}

\section{Discussion}

Epigenetics defined as the structural adaptation of chromosomal regions so as to register, signal or perpetuate altered activity states [18]. There is mounting evidence that epigenetics influences prenatal and postnatal health during pregnancy. Methylation of DNA at the $\mathrm{CpG}$ dinucleotide is the most widely studied epigenetic mechanism in somatic cells. DNA methylation occurs during germ cell and preimplantation development [19,20]. Then studies of investigating the effect of gestational factors that may affect early life development and latent disease risk via changing the 
DNA methylation were performed. Besides, in human sperm, two types of protamines-protamine $1(\mathrm{P} 1)$ and protamine 2 (P2) that are incorporated into the sperm chromatin in equal quantities, are expressed in human sperm cells [5,21]. Aberration of this ratio is associated with male factor infertility, including a reduced sperm count and motility and increased abnormal sperm morphology.

IVF and ICSI are medical treatments that are used to help couples conceive. Fertilization of the oocyte by sperm in vivo and the following uterine transfer of the embryos are involved in these procedures. These initial life experiences of medical treatment offspring are different from the naturally occurring environment. It has been proposed that an abnormal methylation pattern could arise from either a faulty de novo methylation mechanism or the disruption of erasure mechanisms of the existing DNA methylation [22]. The role abnormally methylated sperm genes may play after fertilization is unknown. Recent research investigations demonstrated that environmentally induced parental epigenetic alterations could be passed into the next generations and influence the characteristics of the offspring $[23,24]$. Besides, it was reported by $\mathrm{Yu}$ that histone retention and DNA methylation were altered in density-gradients selected human spermatozoa for assisted reproduction [17]. In the present study, the outcomes of using assisted reproductive technologies with density-gradients or combination with swim-up and swim-up method alone to separate sperm were compared. There were no significant differences in implantation rate, clinical pregnancy rate, miscarriage rate, ectopic pregnancy rate, twin pregnancy rate, still birth rate, prematurity rate, low birth weight rate, macrosomia rate and fetal or baby abnormality rate in two groups. However, the live birth rate of per transferred cycle was higher in the group A than the group B.

Besides, density-gradients selection of sperm can separate sperm with high motility and good morphology. Therefore, sperm separation can effectively increase the overall quality of sperm. It was showed that protamine transition might be increased after sperm separation in semen samples and global DNA methylation might decrease after sperm separation [17]. Such kind of change could be better interpreted as acquiring better-quality sperm after density-gradients separation instead of bad quality of sperm caused by density-gradient methods.

So, we assume that density-gradients (or combination with swim-up method) and swim-up method alone to separate sperm is not the main reason of several epigenetic diseases that are found after assisted reproductive technologies. Both densitygradients (and combination with swim-up method) and swimup method alone to separate sperm are safe to prepare the sperm for IVF and ICSI.

\section{Conflict of Interest}

The authors declare that they have no competing interests.

\section{References}

1. Bashamboo A, Ferraz-de-Souza B, Lourenco D, Lin L, Sebire NJ, Montjean D. Human male infertility associated with mutations in NR5A1 encoding steroidogenic factor 1. Am J Hum Genet 2010; 87: 505-512.

2. Dohle GR, Halley DJ, Van Hemel JO, van den Ouwel AM, Pieters $\mathrm{MH}$, Weber RF. Genetic risk factors in infertile men with severe oligozoospermia and azoospermia. Hum Reprod 2002; 17: 13-16.

3. Erenpreiss J, Spano M, Erenpreisa J, Bungum M, Giwercman A. Sperm chromatin structure and male fertility: biological and clinical aspects. Asian J Androl 2006; 8: 11-29.

4. Aston KI, Krausz C, Laface I, Ruiz-Castane E, Carrell DT. Evaluation of 172 candidate polymorphisms for association with oligozoospermia or azoospermia in a large cohort of men of European descent. Hum Reprod 2010; 25: 1383-1397.

5. Carrell DT, Emery BR, Hammoud S. Altered protamine expression and diminished spermatogenesis: what is the link? Hum Reprod Update 2007; 13: 313-327.

6. Bench GS, Friz AM, Corzett MH, Morse DH, Balhorn R. DNA and total protamine masses in individual sperm from fertile mammalian subjects. Cytometry 1996; 23: 263-271.

7. Monk M, Boubelik M, Lehnert S. Temporal and regional changes in DNA methylation in the embryonic, extraembryonic and germ cell lineages during mouse embryo development. Development 1987; 99: 371-382.

8. Nanassy L, Carrell DT. Abnormal methylation of the promoter of CREM is broadly associated with male factor infertility and poor sperm quality but is improved in sperm selected by density gradient centrifugation. Fertility Sterility 2011; 95: 2310-2314.

9. de Mateo S, Gazquez C, Guimera M, Balasch J, Meistrich ML, Ballesca JL. Protamine 2 precursors (Pre-P2), protamine 1 to protamine 2 ratio (P1/P2), and assisted reproduction outcome. Fertility Sterility 2009; 91: 715-722.

10. Jenkins TG, Aston KI, Meyer TD, Hotaling JM, Shamsi MB, Johnstone EB. Decreased fecundity and sperm DNA methylation patterns. Fertility Sterility 2016; 105: 51-57.

11. Yu B, Qi Y, Liu D, Gao X, Chen H, Bai C. Cigarette smoking is associated with abnormal histone-to-protamine transition in human sperm. Fertility Sterility 2014; 101: 51-57.

12. Hansen M, Kurinczuk JJ, Bower C, Webb S. The risk of major birth defects after intracytoplasmic sperm injection and in vitro fertilization. New England J Med 2002; 346: 725-730.

13. DeBaun MR, Niemitz EL, Feinberg AP. Association of in vitro fertilization with Beckwith-Wiedemann syndrome and epigenetic alterations of LIT1 and H19. Am J Hum Genet 2003; 72: 156-160. 
14. Gicquel C, Gaston V, Mandelbaum J, Siffroi JP, Flahault A, Le Bouc Y. In vitro fertilization may increase the risk of Beckwith-Wiedemann syndrome related to the abnormal imprinting of the KCN1OT gene. Am J Hum Genet 2003; 72: 1338-1341.

15. Kagami M, Nagai T, Fukami M, Yamazawa K, Ogata T. Silver-Russell syndrome in a girl born after in vitro fertilization: partial hypermethylation at the differentially methylated region of PEG1/MEST. J Assisted Reprod Genet 2007; 24: 131-136.

16. Dias BG, Ressler KJ. Parental olfactory experience influences behavior and neural structure in subsequent generations. Nature Neurosci 2014; 17: 89-96.

17. Yu B, Zhou H, Liu M, Zheng T, Jiang L, Zhao M. Epigenetic alterations in density selected human spermatozoa for assisted reproduction. PloS One 2015; 10: $\mathrm{e} 0145585$.

18. Bird A. Perceptions of epigenetics. Nature 2007; 447: 396-398.

19. Smith ZD, Chan MM, Mikkelsen TS, Gu H, Gnirke A, Regev A. A unique regulatory phase of DNA methylation in the early mammalian embryo. Nature 2012; 484: 339-344.

20. Guo H, Zhu P, Yan L, Li R, Hu B, Lian Y. The DNA methylation landscape of human early embryos. Nature 2014; 511: 606-610.
21. Oliva R. Protamines and male infertility. Hum Reprod Update 2006; 12: 417-435.

22. Houshdaran S, Cortessis VK, Siegmund K, Yang A, Laird PW, Sokol RZ. Widespread epigenetic abnormalities suggest a broad DNA methylation erasure defect in abnormal human sperm. PloS One 2007; 2: e1289.

23. Wei Y, Yang CR, Wei YP, Zhao ZA, Hou Y, Schatten H. Paternally induced transgenerational inheritance of susceptibility to diabetes in mammals. Proc Natl Acad Sci U S A 2014; 111: 1873-1878.

24. Liang F, Diao L, Liu J, Jiang N, Zhang J, Wang H. Paternal ethanol exposure and behavioral abnormities in offspring: associated alterations in imprinted gene methylation. Neuropharmacology 2014; 81: 126-133.

\section{*Correspondence to}

Ling Sun

Center of Reproductive Medicine

Guangzhou Women and Children's Medical Center

Guangzhou Medical University

Guangzhou

PR China 